\title{
A Comparison of Outcomes Between Laparoscopic and Robotic Appendectomy Among ACS NSQIP Hospitals
}

Timothy Becker ${ }^{1}$, Kevin Y Pei

Indiana University School of Medicine'; Parkview Health²

\section{Background/Objective:}

Robotic general surgery remains controversial with some employing the technology for common laparoscopic procedures such as appendectomies. Very few studies have compared robotic appendectomies to existing techniques, partly due to the relative scarcity of data. The purpose of this study was to compare outcomes for robotic appendectomies versus laparoscopic appendectomies.

\section{Methods}

This retrospective cohort study evaluated procedural specific databases of ACS-NSQIP for appendectomy between 2016-2019 (inclusive). Demographic and surgical outcomes including composite 30 day complications, specific complications, and length of operation were analyzed using a univariant analysis.

\section{Results}

There was no difference in the total number of comorbidities present or the severity of appendicitis (perforation/abscesses) between robotic and laparoscopic cases. Robotic appendectomy had a longer operation time (91 $\mathrm{min}$ vs $52 \mathrm{~min}, \mathrm{p}<0.001$ ) but a shorter postoperative stay $(0.66$ days vs 1.27 days, $p<0.001)$. There was no difference in the frequency of 30 -day mortality $(p=0.34)$, readmission $(p=0.20)$, or complications $(p=1)$ between robotically performed appendectomy and laparoscopic appendectomy (Table 1)

\begin{tabular}{lllll}
\hline \multirow{2}{*}{ Complications } & Laparoscopic & Robotic & & \\
\cline { 2 - 4 } & $\mathrm{N}=49,800$ & $\mathrm{~N}=50$ & P Value & $\begin{array}{l}\text { Odds } \\
\text { ratio }\end{array}$ \\
\hline Any Complication & $5302(10.6 \%)$ & $5(10 \%)$ & 1 & 1.06 \\
\hline Superficial surgical site infection & $423(0.8 \%)$ & 0 & $>0.9$ & \\
\hline Organ Space SSI & $1,355(2.7 \%)$ & 0 & $>0.9$ & \\
\hline Postoperative Intra-abdominal Abscess & $1353(2.7 \%)$ & 0 & $>0.9$ & \\
\hline Sepsis & $1,696(3.4 \%)$ & $1(2 \%)$ & $>0.9$ & 1.7 \\
\hline
\end{tabular}

Table 1. Complications for Laparoscopic versus Robotic appendectomy. 


\section{Conclusion and Potential Impact}

Our results demonstrated laparoscopic and robotic appendectomy had a similar frequency and profile of complications. Robotic procedures took longer but resulted in shorter post-operative stays. Robotic appendectomies appear promising but at present, only make up a small fraction of cases $(0.1 \%)$ and the widespread adoption of robotic appendectomies is difficult due to issues of cost, equipment, and training. 